\title{
Gene polymorphisms in pattern recognition receptors and susceptibility to idiopathic recurrent vulvovaginal candidiasis
}

\author{
Diana C. Rosentul ${ }^{1,2}$, Corine E. Delsing ${ }^{1,2}$, Martin Jaeger ${ }^{1,2}$, Theo S. Plantinga ${ }^{1,2}$, Marije Oosting $^{1,2}$, \\ Irene Costantini ${ }^{1,2}$, Hanka Venselaar ${ }^{3}$, Leo A. B. Joosten ${ }^{1,2}$, Jos W. M. van der Meer ${ }^{1,2}$, \\ Bertrand Dupont ${ }^{4}$, Bart-Jan Kullberg ${ }^{1,2}$, Jack D. Sobel ${ }^{5,6}$ * and Mihai G. Netea ${ }^{1,2}$ \\ ${ }^{1}$ Department of Internal Medicine, Radboud University Medical Center, Nijmegen, Netherlands \\ ${ }^{2}$ Nijmegen Institute for Infection, Inflammation and Immunity (N4i), Radboud University Medical Center, Nijmegen, Netherlands \\ ${ }^{3}$ Centre for Molecular and Biomolecular Informatics, Nijmegen Center for Molecular Life Science, Radboud University Medical Center, Nijmegen, Netherlands \\ ${ }^{4}$ Hopital Necker-Enfants Malades, Paris, France \\ ${ }^{5}$ Department of Medicine, School of Medicine, Wayne State University, Detroit, MI, USA \\ ${ }^{6}$ Division of Infectious Diseases, Harper University Hospital, Detroit, MI, USA
}

\section{Edited by:}

Evangelos Giamarellos-Bourboulis, University of Athens, Greece

\section{Reviewed by:}

Graeme Forrest, Portland Veteran Affairs Medical Center, USA Evangelos Giamarellos-Bourboulis, University of Athens, Greece

\section{*Correspondence:}

Jack D. Sobel, Division of Infectious Diseases, Harper University Hospital, 3990 John R, Detroit, MI 48201, USA e-mail: jsobel@med.wayne.edu
Objective: Approximately 5\% of women suffer from recurrent vulvovaginal candidiasis (RVVC). It has been hypothesized that genetic factors play an important role in the susceptibility to RVVC. The aim of this study was to assess the effect of genetic variants of genes encoding for pattern recognition receptors (PRRs) on susceptibility to RVVC.

Study design: For the study, 119 RVVC patients and 263 healthy controls were recruited. Prevalence of polymorphisms in five PRRs involved in recognition of Candida were investigated in patients and controls. In silico and functional studies were performed to assess their functional effects.

Results: Single nucleotide polymorphisms (SNPS) in TLR1, TLR4, CLEC7A, and CARD9 did not affect the susceptibility to RVVC. In contrast, a non-synonymous polymorphism in TLR2 (rs5743704, Pro631 His) increased the susceptibility to RVVC almost 3-fold. Furthermore, the TLR2 rs5743704 SNP had deleterious effects on protein function as assessed by in silico analysis, and in vitro functional assays suggested that it reduces production of IL-17 and IFN $\gamma$ upon stimulation of peripheral blood mononuclear cells with Candida albicans. No effects were observed on serum mannose-binding lectin concentrations.

Condensation: This study demonstrates the association of susceptibility to RVVC with genetic variation in TLR2, most likely caused by decreased induction of mucosal antifungal host defense.

Conclusion: Genetic variation in TLR2 may significantly enhance susceptibility to RVVC by modulating host defense mechanisms against Candida. Additional studies are warranted to assess systematically the role of host genetic variation for susceptibility to RVVC.

Keywords: RVVC, genetic variation, pattern recognition receptors, cytokines

\section{INTRODUCTION}

Candida microorganisms, especially Candida albicans, often colonize the genital tract in women, and under certain conditions are responsible for mucosal inflammation (Hurley, 1977; Fleury, 1981; Odds, 1988; Fidel and Sobel, 1996; Giraldo et al., 2000; Sobel, 2007). Vulvovaginal candidiasis (VVC) is a frequent consequence of Candida infection, accompanied by variable pruritus, soreness, rash, and vaginal discharge, with patients experiencing a strong discomfort. Most women have at least one event of VVC in their lifetime, while up to 5-8\% suffer from recurrent vulvovaginal candidiasis (RVVC), defined as at least three infections per year (Hurley, 1977; Sobel, 2007).

Known risk factors of vulvovaginal candidiasis include diabetes, pregnancy, and therapy with glucocorticosteroids, immunosuppressive drugs, and antibiotics (Milsom and Forssman, 1985;
Sobel, 1985; Kent, 1991). However, the vast majority of RVVC patients are healthy women without any identifiable predisposing or episode precipitating factors (Morton, 1977; Sobel, 1985,1993; Kent, 1991; Mardh et al., 2002). Moreover, no distinct C. albicans strains, the species that causes more that $90 \%$ of the VVC episodes, have been described in RVVC patients, arguing against microbiological factors as the major determinants of VVC or susceptibility to recurrent disease (Kent, 1991; Sobel, 1993). Thus, it has been hypothesized that host genetic factors may be a major component determining susceptibility to RVVC.

The innate immune system provides the first barrier against vulvovaginal Candida infection. Pattern recognition receptors (PRRs) on innate immune cells sense molecular moieties on the surface of microorganisms, and thereafter induce an intracellular 
signal that stimulates production of effector molecules such as cytokines or defensins. Two classes of PRRs have been reported to be the main recognition receptors for C. albicans: Toll-like receptors (TLRs) and C-type lectin receptors (CLRs; Netea et al., 2008). TLR4 recognizes fungal cell wall mannans, while TLR2/TLR1 and TLR2/TLR6 heterodimers recognize Candida phospholipomannan (Jouault et al., 2003). Additionally, TLR2 synergizes with DECTIN-1, the receptor for $\beta$-glucan, for the induction of proinflammatory cytokines (Brown et al., 2003; Gantner et al., 2003). DECTIN-1 can also induce TLR-independent signals for the production of IL-17, IL-6, and IL-10 through a Syk/CARD9dependent pathway (Gow et al., 2007). Single nucleotide polymorphisms (SNPs) in both TLRs and CLRs have been described to be associated with an increased susceptibility to both systemic (Plantinga et al., 2012) and oropharyngeal (Plantinga et al., 2010) candidiasis, and we hypothesized that similar effects may be exerted on the susceptibility to RVVC.

The aim of this study was to assess the impact of the SNPs in the genes coding for DECTIN-1 (Tyr238X, rs16910526), CARD9 (Ser12Asn, rs4077515), TLR1 (Arg80Thr, rs5743611), TLR2 (Pro631His, rs5743704), and TLR4 (Asp299Gly, rs4986790; Thr399Ile, rs4986791) on the susceptibility to RVVC.

\section{MATERIALS AND METHODS ETHICS STATEMENT}

The inclusion of patients and controls in this study was approved by the Institutional Review Boards of the Radboud University Medical Centre, School of Medicine, Wayne State University and Hôpital Necker. Enrollment took place between January 2010 and December 2011. Patients gave written informed consent and the study was in accordance to the declaration of Helsinki.

Enrolment of healthy controls for blood donations was approved by the Institutional Review Board of the Radboud University Medical Centre.

\section{PATIENTS AND CONTROL SUBJECTS}

The patient cohort consisting of $119 \mathrm{RVVC}$ patients were recruited at Wayne State University School of Medicine (Detroit, MI, USA), Radboud University Nijmegen Medical Center (Nijmegen, Netherlands) and Hôpital Necker (Paris, France). Patients were enrolled during episodes of acute vaginitis or, if asymptomatic, while receiving maintenance fluconazole therapy. Inclusion criteria for the study were: healthy women above 18 years of age, diagnosed with at least three documented episodes of VVC in a year, microbiologically validated and all caused by C. albicans. Exclusion criteria were use of any immunosuppressive therapy (including steroids), diabetes, pregnancy, and HIV infection. EDTA venous blood was collected after obtaining written informed consent. Asymptomatic healthy controls without a history of vaginal Candida infections $(N=263)$ were recruited at Radboud University Nijmegen Medical Center, Nijmegen, Netherlands and gave written informed consent. All patients and controls were of Western-European genetic background.

\section{GENOTYPING OF THE SINGLE NUCLEOTIDE POLYMORPHISMS}

Genomic DNA was isolated from whole blood using the Qiagen (Valencia, CA, USA) isolation kit and following the standard protocol. The genotype for the CLEC7A (DECTIN1) Tyr328X (rs16910526) and CARD9 Ser12Asn (rs4077515) polymorphisms in the patients was screened by the TaqMan SNP assay C_33748481_10 and C_25956930_20, respectively, (Applied Biosystems, Foster City, CA, USA). The genotype for the TLR1 polymorphism Arg80Thr (rs5743611) was assessed with the TaqMan SNP assay C_27855269_10. The genotype of TLR2 Pro631His (rs5743704) was assessed by applying a predesigned TaqMan SNP assay C_25607736_10. The genotyping for the TLR4 polymorphisms Asp299Gly (rs4986790) and Thr399Ile (rs4986791) was performed with the TaqMan SNP assay C_11722238_20 and C_11722237_20, respectively. The TaqMan qPCR assays were performed on the $7300 \mathrm{ABI}$ Real-Time polymerase chain reaction system (Applied Biosystems). Positive and negative controls were included in the assays.

\section{BIOINFORMATIC ANALYSIS FOR THE TLR2 POLYMORPHISM rs5743704}

The TLR2 protein information was obtained from Swissprot accession code O60603, and OMIM accession code 603028, using the MRS server ${ }^{1}$. We used the PROSITE server ${ }^{2}$ to retrieve information about the TLR2 protein domains. No protein structure is available for the complete TLR2 protein at the moment. However, the cytoplasmic TIR domain is known and can be found as PDBfile $1 \mathrm{O} 77$ (Tao et al., 2002). This structure in this file contains a mutation on position 713 and misses one loop, but the remaining residues are $100 \%$ identical to the sequence of TLR2.

The extracellular domain up to residue 509 is $100 \%$ identical to the protein in PDB file 2Z7X (Jin et al., 2007). The Pro631His polymorphism is located in the region linking the TIR domain with the intracellular domain. We used the automatic modeling script in the YASARA \& WHAT IF Twinset (Krieger et al., 2002) to extend the known structures and to model missing loops in these structures. As a result, we produced models of the $\mathrm{N}$ - and of the C-terminal domain, which are both accurate, because they are based on the known structure of that protein. These structures are extended with residues of the linking region. Additionally, we used $\mathrm{HOPE}^{3}$, a next generation bioinformatics web server that performs automatic mutant analysis (Venselaar et al., 2010). Besides this, an analysis of the pathogenicity of the Pro631His polymorphism in TLR2 was performed using conservation and structural information. The Polyphen-2, SIFT, PANTHER, snps3D, and the SNAP servers where employed for this analysis.

\section{CYTOKINE STIMULATION ASSAYS}

Peripheral blood mononuclear cells (PBMCs) were isolated from 73 healthy volunteers by Ficoll-Paque gradient. All healthy volunteers gave written informed consent. $0.5 \times 10^{6} \mathrm{PBMCs} /$ well were plated in round bottom wells plates. Subsequently, incubation with C. albicans blastoconidia $\left(1 \times 10^{6} / \mathrm{mL}\right.$, heat-killed at $100^{\circ} \mathrm{C}$ for $1 \mathrm{~h}$ ) was performed for $24 \mathrm{~h}, 48 \mathrm{~h}$ or 7 days. The incubation time varied for each cytokine: $24 \mathrm{~h}$ for IL- $1 \beta$ and IL- $6,48 \mathrm{~h}$ for IFN $\gamma$ and IL-10, and 7 days for IL-17 (with the addition of $10 \%$ human serum). After the incubation time, cytokines were

\footnotetext{
${ }^{1}$ http://mrs.cmbi.ru.nl/mrs-web

${ }^{2}$ http://expasy.org/prosite

${ }^{3}$ http://www.cmbi.ru.nl/hope
} 
measured in the supernatants by ELISA (R\&D Systems, MN, USA or Sanquin Research, Amsterdam, Netherlands). Detection limits were $40 \mathrm{pg} / \mathrm{mL}$, except for the IFN $\gamma$ ELISA (12 pg/mL). Mannosebinding lectin $(\mathrm{MBL})$ concentrations in serum were measured by ELISA (Bioporto, Gentofte, Denmark).

\section{STATISTICAL ANALYSIS}

For the analysis of the polymorphisms in the PRRs, statistical comparisons of frequencies were made between RVVC versus control subjects. We used SPSS 16.0 software to perform Chi-square tests and the 5\% Confidence Intervals Odds ratio to calculate the risk. Furthermore, for the analysis of the effect of the TLR2 genotype on the in vitro cytokine production, a non-parametrical Kruskal-Wallis analysis was performed, and for the circulatory MBL measurement we used the Mann-Whitney $U$-test. Both analyses were done using GraphPad Prism version 4.00 for Windows, GraphPad Software, San Diego, CA, USA.

\section{RESULTS}

\section{THE INFLUENCE OF POLYMORPHISMS IN PRR GENES ON THE} SUSCEPTIBILITY TO RVVC

As a polymorphism in the gene encoding the CLR DECTIN1 (CLEC7A) has previously been shown to be associated with an increased susceptibility to fungal infections, a first set of comparisons assessed the prevalence of CLEC7A and CARD9 polymorphisms in control individuals and patients with RVVC (Table 1). No effects of CLEC7A (Tyr238X, rs16910526) and CARD9 (Ser12Asn, rs4077515) polymorphisms on the susceptibility to RVVC have been observed. In a second set of experiments, the prevalence of polymorphisms in the genes coding for TLR1 (Arg80Thr, rs5743611), TLR2 (Pro631His, rs5743704), and TLR4 (Asp299Gly, rs4986790; Thr399Ile, rs4986791) was also assessed.
These analyses revealed that the polymorphisms in the TLR 1 and TLR4 genes did not affect the susceptibility to RVVC. In contrast, the genotype for the Pro631His polymorphism on the TLR2 gene was associated with a 2.705 -fold increase $(P$-value 0.046$)$ in susceptibility to RVVC in the patient cohort. All genetic distributions are in Hardy-Weinberg equilibrium (Table 1).

\section{BIO-INFORMATIC ANALYSIS TLR2 rs5743704 POLYMORPHISM}

Because the TLR2 Pro631His polymorphism was associated with an increased susceptibility to RVVC, the functional effects of this genetic variation were studied. In a first analysis, an in silico evaluation of the effect of the Pro631His polymorphism on the TLR2 protein was performed. We evaluated its effect at the genomic level, by performing multiple sequence alignment of the homologous DNA sequences from 35 other species via the Ensembl server. The cytosine base is conserved at the chromosome 4 nucleotide position 154625451, being also the ancestral allele, evolutionary conservation that underlines a high likelihood that this position in the molecule is functionally important.

To analyze the effects of the SNP rs5743704 on the TLR2 protein, we analyzed the possible domains identified via the Prosite server. The amino acid change for the Pro631His polymorphism affects an amino acid residue that is located near the TIR domain (residue 639-784). To visualize the Pro631His polymorphism on the 3D structure of the TIR domain, we evaluated the homology model using the YASARA software. A screenshot of the 3D homology model is shown in Figure 1. The amino acid change is situated between the intracellular domain and the TIR domain, and might introduce a loss in rigidity when substituting proline by histidine.

Finally, the HOPE server predicted that due to the fact that the polymorphism introduces an amino acid with different

Table 1 | Genetic association of recurrent vulvovaginal candidiasis (RVVC) with polymorphisms in DECTIN-1, CARD9, TLR1, TLR2, and TLR4 genes.

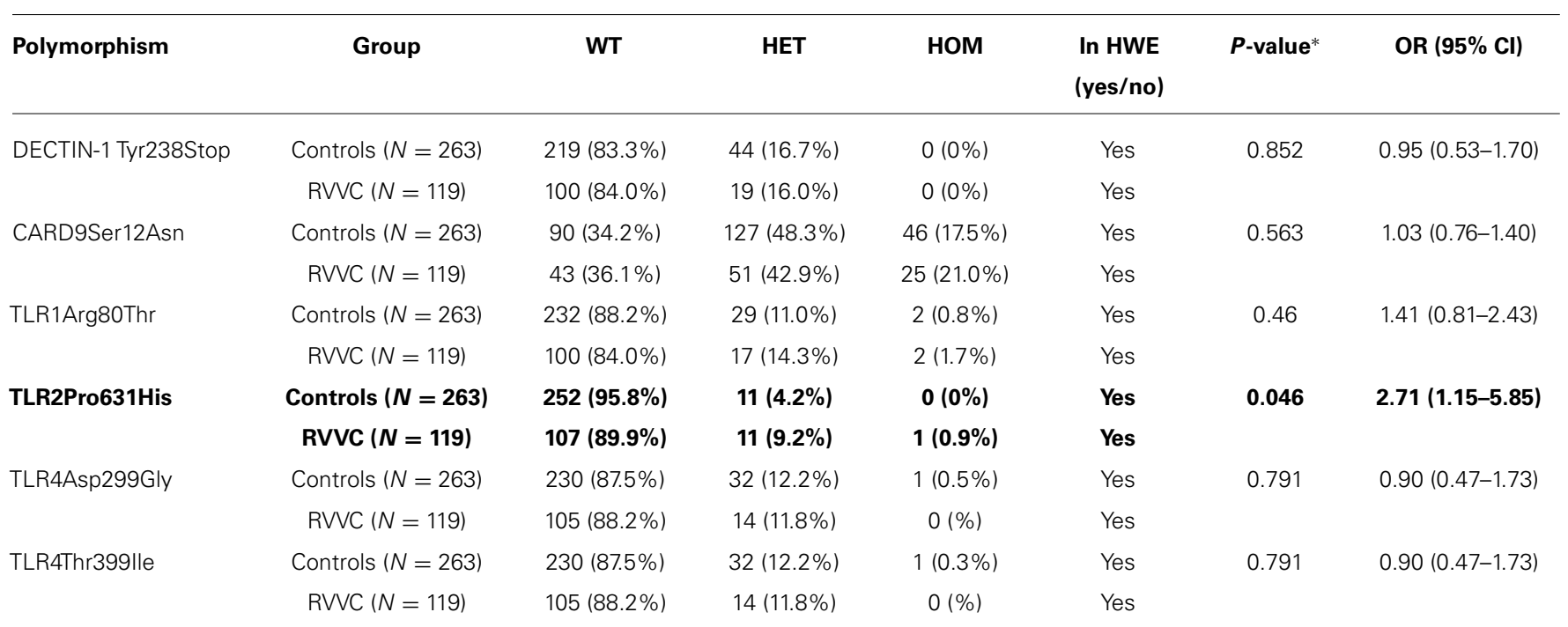

Data are presented as number of individuals (\% major or minor allele), P-value and OR (95\% Cl).

Bold indicates statistically significant association.

${ }^{*}$ Chi-square $\left(\chi^{2}\right)$ test. WT, wild-type; HET, heterozygous; HOM, homozygous; HWE, Hardy-Weinberg equilibrium. 
characteristics, this would affect the function of the molecule. The wild-type residue proline is rigid, resulting in a characteristic backbone conformation that might be required at this position. Additionally, proline is more hydrophobic than histidine, and this may also influence the function of the molecule through loss of hydrophobic interactions on the surface of the protein. This hypothesis was also tested through a series of web servers to predict the pathogenicity of the Pro631His amino acid change. As shown in Supplementary Table 1, according to the Polyphen2, SIFT, PANTHER, and snps3D, the effect of the Pro631His polymorphism is predicted detrimental for TLR2 function.

\section{THE EFFECT OF THE TLR2 Pro631His POLYMORPHISM ON THE PRODUCTION OF INFLAMMATORY MEDIATORS}

In order to assess the functional consequences of the TLR2 Pro631His polymorphism in further detail, cytokine production of PBMCs stimulated with heat-killed C. albicans was assessed in a group of healthy volunteers. The major limitation of this part of the study was that only two individuals in this group were heterozygous for the variant allele, which precludes drawing definitive conclusions. However, we observed that whereas the IL- $1 \beta$, IL6 , and $\mathrm{TNF} \alpha$ production was in the same range comparing the different genotype groups, both individuals bearing the mutated allele displayed a strongly diminished release of the T-cell derived cytokines IFN $\gamma$ and IL-17 (Figure 2), known to be important for antifungal host defense (Pandiyan et al., 2011; van de Veerdonk et al., 2011; Johnson et al., 2012). In contrast, MBL circulating concentrations in the RVVC patients did not differ depending on the TLR2 genotype (Figure 3).

\section{DISCUSSION}

Vulvovaginal candidiasis is one of the most common infections in women. Despite known risk factors such as the hormonal status, diabetes, pregnancy or immunosuppressive therapy (Milsom and Forssman, 1985; Sobel, 1985,1993; Kent, 1991; Nawrot et al., 2000), the majority of the patients are healthy women who are not exposed to these conditions. In the present study, we

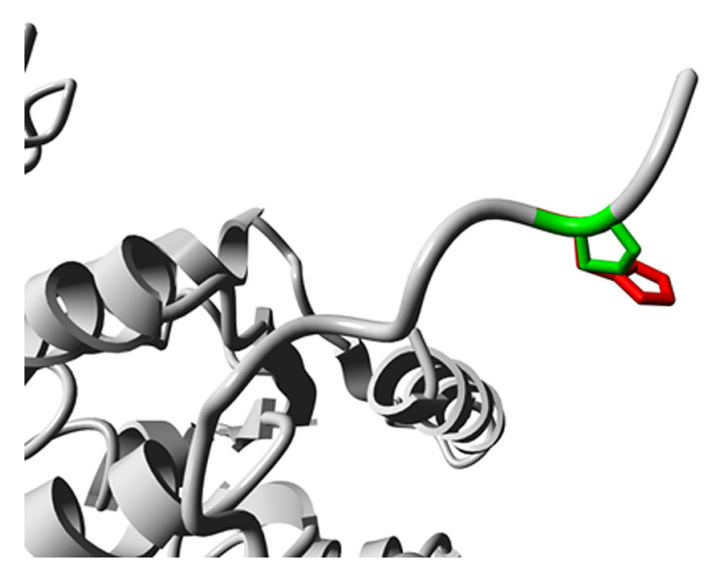

FIGURE 1 | Screenshot of the 3D homology model of the TLR2 protein using the YASARA software. The side chains of the wild-type and mutant residues are shown in green and red, respectively. explored whether genetic variation in genes coding for PRRs involved in Candida recognition (Jouault et al., 2003) affects susceptibility to RVVC. We show that a non-synonymous polymorphism in TLR2 is associated with increased susceptibility to RVVC. In silico assessment suggested functional consequences of the TLR2 polymorphism, and this hypothesis is supported by the decreased production of T-cell derived cytokines IFN $\gamma$ and IL-17 in two individuals bearing the mutation. However, a definitive conclusion was not able to be drawn due to the low number of volunteers with TLR2 SNP identified in the functional studies.

Genetic variants in PRRs have been previously reported to influence predisposition to RVVC. This has been described in a family of patients with a complete deficiency of DECTIN-1, due to an early stop codon mutation in the CLEC7A gene coding for the DECTIN-1 receptor (Ferwerda et al., 2009). Functional consequences of DECTIN-1 deficiency were demonstrated to include impaired induction of both innate and adaptive Th17 immune responses. Furthermore, several other studies have shown the involvement of adaptive immunity in RVVC in humans (Babula et al., 2005; De Luca et al., 2013). In addition to RVVC occurrence in two of the three patients with DECTIN-1 deficiencies, all suffered from onychomycosis. This mutation appears to be relatively common, up to $15 \%$ heterozygosity in populations of European origin, allowing epidemiological studies in heterozygous patients (Ferwerda et al., 2009). Moreover, heterozygosity in the CLEC7A gene is associated with increased mucosal Candida colonization (Plantinga et al., 2009). Thus, we assessed its impact in the present study. The loss of one functional allele, however, does not seem to have a major impact on the susceptibility to RVVC. This implies that only a complete loss-of-function in the molecule is associated with a significant risk for infection. In addition, it has also recently been shown that this polymorphism does not affect the predisposition to disseminated Candida infections (Plantinga et al., 2009; Rosentul et al., 2011).

CARD9 is an adaptor molecule mediating signals induced by DECTIN-1, but also other CLRs. Its role in antifungal immunity has been demonstrated both in knock-out mouse studies (Gross et al., 2006), as well as by the increased susceptibility to severe fungal infections in patients defective in this molecule due to an early stop codon in position Gln295Stop (Glocker et al., 2009). However, this mutation is rare and has not been reported in other individuals. The evaluation of the effects of a known CARD9 Ser12Asn polymorphism did not yield a significant association with RVVC susceptibility in the present study. Similarly, we have previously shown that this polymorphism does not influence the susceptibility to candidemia (Rosentul et al., 2011).

In addition to CLRs, TLRs are the second major family of PRRs involved in the recognition of fungi in general, and Candida in particular, TLR2, TLR4, and TLR1 being most important in antifungal immunity (Netea and Marodi, 2010). While TLR1 polymorphisms have been recently shown to influence susceptibility to candidemia (Plantinga et al., 2012), no such effect was apparent in patients with RVVC. Similarly, two TLR4 polymorphisms that have been previously associated with susceptibility to Gram-negative sepsis and other infections did not 


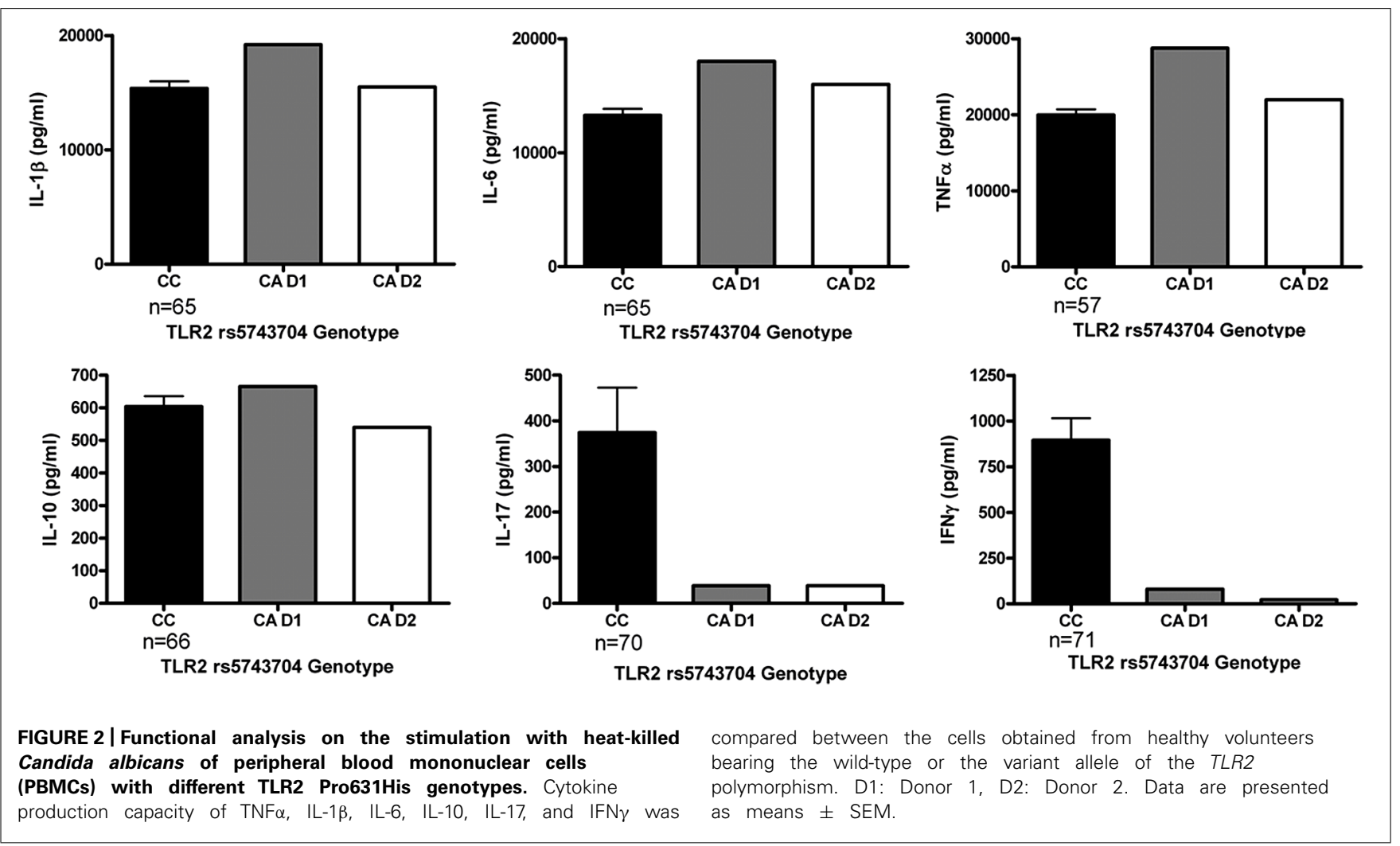

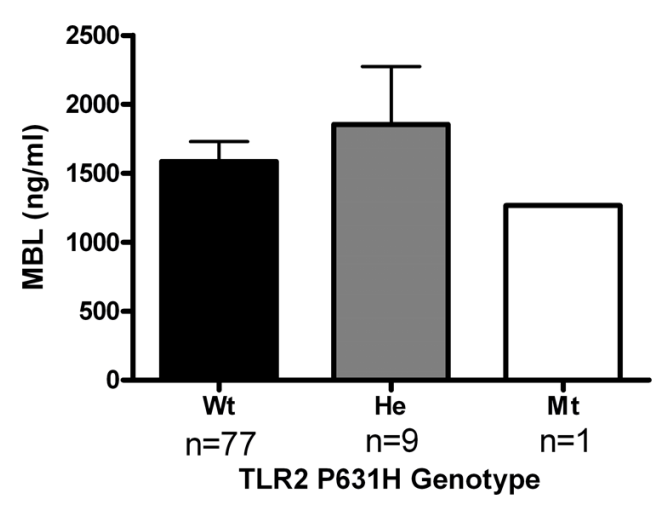

FIGURE 3 | Analysis on the mannose-binding lectin (MBL) concentrations in serum obtained from RVVC patients bearing the wild-type or the variant allele of the TLR2 polymorphism Pro631His. Wt, wild-type; He, heterozygous. Data are presented as means \pm SEM.

have a significant effect on susceptibility to RVVC (Schroder and Schumann, 2005).

In contrast, the TLR2 Pro631His polymorphism induced an almost threefold increase in the susceptibility to RVVC. TLR2 is an important PRR for C. albicans recognition, activating innate immune responses both alone and in synergy with DECTIN-1 (Ferwerda et al., 2008). The deficiency of TLR2 influences susceptibility to systemic candidiasis in mice (Netea et al., 2004), but no studies have been performed in vaginal candidiasis models. The in silico analysis using homology modeling and conservation analysis suggests detrimental effects of the mutation on the function of the receptor. This is supported by the study of Etokebe et al. (2010) that the Pro631His polymorphism has a dominant negative effect on the TLR2 signaling in HEK-293T cells. Finally, we studied the functional relevance of this polymorphism in primary cells from individuals bearing the various alleles. Although we were able to assess cytokine production in only two individuals with a mutant TLR2 allele, both of them consistently produced very low amounts of the T-cell derived cytokines IFN $\gamma$ and IL-17, mediators that are crucial for mucosal antifungal defense (Ferwerda et al., 2009; van de Veerdonk et al., 2011). This observation is supported by the finding of Ben-Ali et al. (2011) who demonstrated that the $631 \mathrm{His}$ $T L R 2$ variant leads to reduced $N F-\kappa B$ activation.

In addition to the effects of the TLR 2 polymorphism on cytokine production, we have also assessed its influence on the concentrations of MBL. MBL is a circulating receptor for mannose residues which activates the complement system and opsonizes Candida (Babula et al., 2005). MBL deficiency is associated with an increased susceptibility to RVVC (Babula et al., 2003; Kaoro Horie Wojitani etal., 2012). However, no differences in serum MBL concentrations were found between individuals bearing different TLR2 alleles, showing that the effect of the TLR2 Pro631His polymorphism on the RVVC susceptibility is independent of the production of MBL. Ideally, the cytokine analyses should be extended to assessment in lavage fluids or cells. However, these materials were not collected from the patients presented in this manuscript, and future studies should address this aspect.

In conclusion, in this study we provide evidence that polymorphisms in PRRs may play an important role in susceptibility 
to RVVC in otherwise healthy women. While CLEC7A, CARD9, TLR1, and TLR4 polymorphisms had no impact, the TLR2 Pro631His polymorphism was associated with an almost 3-fold increase in susceptibility to RVVC. These data demonstrate the role of TLR2 genetic variation in innate immunity genes for RVVC, and future investigations are warranted in larger cohorts if possible to replicate and extend the results of the present study.

\section{ACKNOWLEDGMENTS}

Diana C. Rosentul was funded by the European Commission through the FINSysB Marie Curie Initial Training Network (PITNGA-2008-214004). Theo S. Plantinga was supported by a Veni grant of the Netherlands Organization for Scientific Research (NWO). Mihai G. Netea was supported by a European Research Council Consolidator Grant (ERC-StG-310372). All authors had full access to all of the data in the study and take responsibility for the integrity of the data and the accuracy of the data analysis.

\section{SUPPLEMENTARY MATERIAL}

The Supplementary Material for this article can be found online at: http://www.frontiersin.org/journal/10.3389/fmicb.2014.00483/ abstract

\section{REFERENCES}

Adzhubei, I. A., Schmidt, S., Peshkin, L., Ramensky, V. E., Gerasimova, A., Bork, P., et al. (2010). A method and server for predicting damaging missense mutations. Nat. Methods 7, 248-249. doi: 10.1038/nmeth0410-248

Babula, O., Lazdane, G., Kroica, J., Ledger, W. J., and Witkin, S. S. (2003). Relation between recurrent vulvovaginal candidiasis, vaginal concentrations of mannosebinding lectin, and a mannose-binding lectin gene polymorphism in latvian women. Clin. Infect. Dis. 37, 733-737. doi: 10.1086/377234

Babula, O., Lazdane, G., Kroica, J., Linhares, I. M., Ledger, W. J., and Witkin, S. S. (2005). Frequency of interleukin-4 (IL-4) -589 gene polymorphism and vaginal concentrations of IL-4, nitric oxide, and mannose-binding lectin in women with recurrent vulvovaginal candidiasis. Clin. Infect. Dis. 40, 1258-1262. doi: $10.1086 / 429246$

Ben-Ali, M., Corre, B., Manry, J., Barreiro, L. B., Quach, H., Boniotto, M., et al. (2011). Functional characterization of naturally occurring genetic variants in the human TLR1-2-6 gene family. Hum. Mutat. 32, 643-652. doi: 10.1002/humu.21486

Bromberg, Y., and Rost, B. (2007). SNAP: predict effect of non-synonymous polymorphisms on function. Nucleic Acids Res. 35, 3823-3835. doi: 10.1093/nar/gkm238

Brown, G. D., Herre, J., Williams, D. L., Willment, J. A., Marshall, A. S., and Gordon, S. (2003). Dectin-1 mediates the biological effects of beta-glucans. J. Exp. Med. 197, 1119-1124. doi: 10.1084/jem.20021890

De Luca, A., Carvalho, A., Cunha, C., Iannitti, R. G., and Pitzurra, L. (2013). IL-22 and IDO1 affect immunity and tolerance to murine and human vaginal candidiasis. PLoS Pathog. 9:e1003486. doi: 10.1371/journal.ppat.1003486

Etokebe, G. E., Skjeldal, F., Nilsen, N., Rodionov, D., Knezevic, J., Bulat-Kardum, L., et al. (2010). Toll-like receptor $2(\mathrm{P} 631 \mathrm{H})$ mutant impairs membrane internalization and is a dominant negative allele. Scand. J. Immunol. 71, 369-381. doi: 10.1111/j.1365-3083.2010.02379.x

Ferwerda, B., Ferwerda, G., Plantinga, T. S., Willment, J. A., van Spriel, A. B., Venselaar, H., et al. (2009). Human dectin-1 deficiency and mucocutaneous fungal infections. N. Engl. J. Med. 361, 1760-1767. doi: 10.1056/NEJMoa0901053

Ferwerda, G., Meyer-Wentrup, F., Kullberg, B. J., Netea, M. G., and Adema, G. J. (2008). Dectin-1 synergizes with TLR2 and TLR4 for cytokine production in human primary monocytes and macrophages. Cell. Microbiol. 10, 2058-2066. doi: $10.1111 / \mathrm{j} .1462-5822.2008 .01188 . \mathrm{x}$

Fidel, P. L., and Sobel, J. D. (1996). Immunopathogenesis of recurrent vulvovaginal candidiasis. Clin. Microbiol. Rev. 9, 335-348.

Fleury, F. J. (1981). Adult vaginitis. Clin. Obstet. Gynecol. 24, 407-438. doi: 10.1097/00003081-198106000-00008
Gantner, B. N., Simmons, R. M., Canavera, S. J., Akira, S., and Underhill, D. M. (2003). Collaborative induction of inflammatory responses by dectin-1 and toll-like receptor 2. J. Exp. Med. 197, 1107-1117. doi: 10.1084/jem.20021787

Giraldo, P., von Nowaskonski, A., Gomes, F. A. M., Linhares, I., Neves, N. A., and Witkin, S. S. (2000). Vaginal colonization by Candida in asymptomatic women with and without a history of recurrent vulvovaginal candidiasis. Obstet. Gynecol. 95, 413-416. doi: 10.1016/S0029-7844(99)00577-3

Glocker, E. O., Hennigs, A., Nabavi, M., Schaffer, A. A., Woellner, C., Salzer, U., et al. (2009). A homozygous CARD9 mutation in a family with susceptibility to fungal infections. N. Eng. J. Med. 361, 1727-1735. doi: 10.1056/NEJMoa0810719

Gow, N. A. R., Netea, M. G., Munro, C. A., Ferwerda, G., Bates, S., and MoraMontes, H. M., et al. (2007). Immune recognition of Candida albicans $\beta$-glucan by dectin-1. J. Infect. Dis. 196, 1565-1571. doi: 10.1086/523110

Gross, O., Gewies, A., Finger, K., Schafer, M., Sparwasser, T., Peschel, C., et al. (2006). Card 9 controls a non-TLR signalling pathway for innate anti-fungal immunity. Nature 442, 651-656. doi: 10.1038/nature04926

Hurley, R. (1977). Candidal vaginitis. Proc. R. Soc. Med. 70, 1-3.

Jin, M. S., Kim, S. E., Heo, J. Y., Lee, M. E., Kim, H. M., Paik, S. G., et al. (2007). Crystal structure of the TLR1-TLR2 heterodimer induced by binding of a tri-acylated lipopeptide. Cell 130, 1071-1082. doi: 10.1016/j.cell.2007.09.008

Johnson, M. D., Plantinga, T. S., van de Vosse, E., Velez Edwards, D. R., Smith, P. B., Alexander, B. D., et al. (2012). Cytokine gene polymorphisms and the outcome of invasive candidiasis: a prospective cohort study. Clin. Infect. Dis. 54, 502-510. doi: $10.1093 / \mathrm{cid} / \mathrm{cir} 827$

Jouault, T., Ibata-Ombetta, S., Takeuchi, O., Trinel, P. A., Sacchetti, P., and Lefebvre, P. (2003). Candida albicans phospholipomannan is sensed through toll-like receptors. J. Infect. Dis. 188, 165-172. doi: 10.1086/375784

Kaoro Horie Wojitani, M. D., de Aguiar, L. M., Baracat, E. C., and Linhares, I. M. (2012). Association between mannose-binding lectin and interleukin-1 receptor antagonist gene polymorphisms and recurrent vulvovaginal candidiasis. Arch. Gynecol. Obstet. 285, 149-153. doi: 10.1007/s00404-011-1920-z

Kent, H. L. (1991). Epidemiologu of vaginitis. Am. J. Obstet. Gynecol. 165, 11681176. doi: 10.1016/S0002-9378(12)90722-X

Krieger, E., Koraimann, G., and Vriend, G. (2002). Increasing the precision of comparative models with YASARA NOVA - a self-parameterizing force field. Proteins 47, 393-402. doi: 10.1002/prot.10104

Mardh, P. A., Rodrigues, A. G., Genc, M., Novikova, N., Martinez-de-Oliveira, J., and Guaschino, S. (2002). Facts and myths on recurrent vulvovaginal candidosis - a review on epidemiology, clinical manifestations, diagnosis, pathogenesis and therapy. Int. J. STD AIDS 13, 522-539. doi: 10.1258/095646202760159639

Milsom, I., and Forssman, L. (1985). Repeated candidiasis - reinfection or recrudescence - A review. Am. J. Obstet. Gynecol. 52, 956-959. doi: 10.1016/S00029378(85)80009-0

Morton, R. S. (1977). Candidal vaginitis - natural-history, predisposing factors and prevention. Proc. R. Soc. Med. 70(Suppl. 4), 3-6.

Nawrot, U., Grzybek-Hryncewicz, K., Zielska, U., Czarny, A., and Podwinska, J. (2000). The study of cell-mediated immune response in recurrent vulvovaginal candidiasis. FEMS Immunol. Med. Microbiol. 29, 89-94. doi: 10.1111/j.1574695X.2000.tb01509.x

Netea, M. G., Brown, G. D., Kullberg, B. J., and Gow, N. A. R. (2008). An integrated model of the recognition of Candida albicans by the innate immune system. Nat. Rev. Microbiol. 6, 67-78. doi: 10.1038/nrmicro1815

Netea, M. G., and Marodi, L. (2010). Innate immune mechanisms for recognition and uptake of Candida species. Trends Immunol. 31, 346-353. doi: 10.1016/j.it.2010.06.007

Netea, M. G., Sutmuller, R., Hermann, C., Van der Graaf, C. A., Van der Meer, J. W., van Krieken, J. H., et al. (2004). Toll-like receptor 2 suppresses immunity against Candida albicans through induction of IL-10 and regulatory T cells. J. Immunol. 172, 3712-3718. doi: 10.4049/jimmunol.172.6.3712

Ng, P. C., and Henikoff, S. (2003). SIFT: predicting amino acid changes that affect protein function. Nucleic Acids Res. 31, 3812-3814. doi: 10.1093/nar/gkg509

Odds, F. C. (1988). "Chronic mucocutaneous candidiosis," in Candida and Candidiasis, ed. F. C. Odds (Baltimore, MD: University Park Press), 104-110.

Pandiyan, P., Conti, H. R., Zheng, L., Peterson, A. C., Mathern, D. R., HernándezSantos, N., et al. (2011). CD4(+)CD25(+)Foxp3(+) regulatory T cells promote Th17 cells in vitro and enhance host resistance in mouse Candida albicans Th17 cell infection model. Immunity 34, 422-434. doi: 10.1016/j.immuni.2011. 03.002 
Plantinga, T. S., Hamza, O. J. M., Willment, J. A., Ferwerda, B., van de Geer, N. M., Verweij, P. E., et al. (2010). Genetic variation of innate immune genes in HIV-infected african patients with or without oropharyngeal candidiasis. $J$. Acquir. Immune. Defic. Syndr. 55, 87-94. doi: 10.1097/QAI.0b013e3181e53c64

Plantinga, T. S., Johnson, M. D., Scott, W. K., van de Vosse, E., Velez Edwards, D. R., Smith, P. B., et al. (2012). Toll-like receptor 1 polymorphisms increase susceptibility to candidemia. J. Infect. Dis. 205, 934-943. doi: 10.1093/infdis/jir867

Plantinga, T. S., van der Velden, W., Ferwerda, B., van Spriel, A. B., Adema, G., Feuth, T., et al. (2009). Early stop polymorphism in human DECTIN-1 is associated with increased Candida colonization in hematopoietic stem cell transplant recipients. Clin. Infect. Dis. 49, 724-732. doi: 10.1086/604714

Rosentul, D. C., Plantinga, T. S., Oosting, M., Scott, W. K., Velez Edwards, D. R., Smith, P. B., et al. (2011). Genetic variation in the dectin-1/CARD9 recognition pathway and susceptibility to candidemia. J. Infect. Dis. 204, 1138-1145. doi: 10.1093/infdis/jir458

Schroder, N. W., and Schumann, R. R. (2005). Single nucleotide polymorphisms of Toll-like receptors and susceptibility to infectious disease. Lancet Infect. Dis. 5, 156-164. doi: 10.1016/S1473-3099(05)01308-3

Sobel, J. D. (1985). Epidemiology and pathogenesis of recurrent vulvovaginal candidiasis. Am. J. Obstet. Gynecol. 152, 924-935. doi: 10.1016/S00029378(85)80003-X

Sobel, J. D. (1993). Candidal vulvo-vaginitis. Clin. Obstet. Gynecol. 36, 153-165. doi: 10.1097/00003081-199303000-00021

Sobel, J. D. (2007). Vulvovaginal candidosis. Lancet 369, 1961-1971. doi: 10.1016/S0140-6736(07)60917-9

Tao, X., Xu, Y., Zheng, Y., Beg, A. A. and Tong, L. (2002). An extensively associated dimer in the structure of the C713S mutant of the TIR domain of human TLR2. Biochem. Biophys. Res. Commun. 299, 216-221. doi: 10.1016/S0006-291X(02)02581-0

Thomas, P. D., Campbell, M. J., Kejariwal, A., Mi, H., Karlak, B., Diemer, K., et al. (2003). PANTHER: a library of protein families and subfamilies indexed by function. Genome Res. 13, 2129-2141. doi: 10.1101/gr.772403 van de Veerdonk, F. L., Plantinga, T. S., Hoischen, A., Smeekens, S. P., Joosten L. A., Gilissen, C., etal. (2011). STAT1 mutations in autosomal dominant chronic mucocutaneous candidiasis. N. Eng. J. Med. 365, 54-61. doi: 10.1056/NEJMoa1100102

Venselaar, H., Te Beek, T. A. K. R., Hekkelman, M. L., and Vriend, G. (2010). Protein structure analysis of mutations causing inheritable diseases. An e-Science approach with life scientist friendly interfaces. BMC Bioinformatics 11:548. doi: 10.1186/1471-2105-11-548

Yue, P., Melamud, E., and Moult, J. (2006). SNPs3D: candidate gene and SNP selection for association studies. BMC Bioinformatics 7:166. doi: 10.1186/14712105-7-166

Conflict of Interest Statement: The authors declare that the research was conducted in the absence of any commercial or financial relationships that could be construed as a potential conflict of interest.

Received: 05 August 2014; accepted: 28 August 2014; published online: 23 September 2014.

Citation: Rosentul DC, Delsing CE, Jaeger M, Plantinga TS, Oosting M, Costantini I, Venselaar H, Joosten LAB, van der Meer JWM, Dupont B, Kullberg B-J, Sobel JD and Netea MG (2014) Gene polymorphisms in pattern recognition receptors and susceptibility to idiopathic recurrent vulvovaginal candidiasis. Front. Microbiol. 5:483. doi: 10.3389/fmicb.2014.00483

This article was submitted to Infectious Diseases, a section of the journal Frontiers in Microbiology.

Copyright (c) 2014 Rosentul, Delsing, Jaeger, Plantinga, Oosting, Costantini, Venselaar, Joosten, van der Meer, Dupont, Kullberg, Sobel and Netea. This is an open-access article distributed under the terms of the Creative Commons Attribution License (CC BY). The use, distribution or reproduction in other forums is permitted, provided the original author(s) or licensor are credited and that the original publication in this journal is cited, in accordance with accepted academic practice. No use, distribution or reproduction is permitted which does not comply with these terms. 